\title{
Clinical Characteristics and Severity of COVID-19 Disease in Patients from Boston Area Hospitals
}

Hesam Dashti, Ph.D.

Division of Preventive Medicine, Brigham and Women's Hospital and Harvard Medical School, Boston,

MA, USA

David W. Bates, B.S.

Division of Preventive Medicine, Brigham and Women's Hospital, USA

Elise Roche, B.A.

Division of Preventive Medicine, Brigham and Women's Hospital, Boston, MA, USA

Julie Fiskio, B.S.

Information Systems, Partners HealthCare System, Wellesley, MA

Samia Mora*, M.D., M.H.S.

Division of Preventive Medicine, Brigham and Women's Hospital, Boston, MA, USA

Division of Cardiovascular Medicine, Brigham and Women's Hospital and Harvard Medical School,

Boston, MA, USA

Olga V. Demler*, Ph.D.

Division of Preventive Medicine, Brigham and Women's Hospital and Harvard Medical School, Boston,

MA, USA

*contributed equally

Samia Mora,

Division of Preventive Medicine,

Brigham and Women's Hospital,

900 Commonwealth Ave,

Boston, MA, USA 02115 
medRxiv preprint doi: https://doi.org/10.1101/2020.07.27.20163071; this version posted August 4, 2020. The copyright holder for this preprint (which was not certified by peer review) is the author/funder, who has granted medRxiv a license to display the preprint in perpetuity.

All rights reserved. No reuse allowed without permission.

Olga V. Demler,

Division of Preventive Medicine,

Brigham and Women's Hospital,

900 Commonwealth Ave,

Boston, MA, USA 02115

Word Count:

Abstract 97

Manuscript 575 
medRxiv preprint doi: https://doi.org/10.1101/2020.07.27.20163071; this version posted August 4, 2020. The copyright holder for this preprint (which was not certified by peer review) is the author/funder, who has granted medRxiv a license to display the preprint in perpetuity.

All rights reserved. No reuse allowed without permission.

\author{
Abstract \\ We summarize key demographic, clinical, and medical characteristics of patients with respect to the \\ severity of COVID-19 disease using Electronic Health Records Data of 4,140 SARS-CoV-2 positive subjects \\ from several large Boston Area Hospitals. We found that prior use of antihypertensive medications as \\ well as lipid lowering and other cardiovascular drugs (such as direct oral anticoagulants and \\ antiplatelets) all track with increased severity of COVID-19 and should be further investigated with \\ appropriate adjustment for confounders such as age and frailty. The three most common prior \\ comorbidities are hyperlipidemia, hypertension, and prior pneumonia, all associated with increased \\ severity.
}

COVID-19, SARS-CoV-2, medication use, comorbid conditions

\title{
Background
}

The incidence of COVID-19 in Massachusetts has been near exponential, ranking third nationwide with more than 66,000 confirmed case. Partners HealthCare, which includes several large Boston-area hospitals, has reported 4,140 COVID-19 patients as of April 18, 2020. We summarize key demographic, clinical, and medical characteristics of these patients with respect to the severity of COVID-19 disease.

\section{Methods}

The Partners Electronic Health Record (EHR) contains outpatient and inpatient records for $>4$ million patients mostly from greater Boston, Massachusetts. The patient population in this study includes all patients $(N=4,140)$ with at least one positive or presumptive positive COVID-19 test result ${ }^{1}$ documented in the Partners EHR between December 1, 2019 and April 18, 2020. The study was approved by the Partners Institutional Review Board. Laboratory, clinical, and medical records (April 1, 2015-April 18, 2020) were obtained from the Partners Research Patients Data Registry (RPDR) and the Enterprise Data Warehouse $(E D W)^{2-4}$. All data was updated daily, except for prior conditions which are updated annually 
medRxiv preprint doi: https://doi.org/10.1101/2020.07.27.20163071; this version posted August 4, 2020. The copyright holder for this preprint (which was not certified by peer review) is the author/funder, who has granted medRxiv a license to display the preprint in perpetuity.

All rights reserved. No reuse allowed without permission.

using a machine learning algorithm ${ }^{4}$. Income data was linked to the 2018 US Census. Medication use was coded as ever in 2015-2020. Severity was defined in five mutually exclusive categories (I) outpatients only, (II) evaluation in an emergency department without admission, (III) inpatient admission not requiring intensive care unit (ICU) care, (IV) inpatient admission requiring ICU care, and (V) deceased. Two-sided tests of trend were performed unless otherwise noted. Python and R software languages were used. All data were obtained from Electronic Health Records Repository Maintained by Brigham Mass General HealthCare Systems in full compliance with the IRB protocols and met all data access requirements. Obtaining consent from all individuals would make the study challenging logistically, financially and scientifically, especially due to the urgent nature of the pandemic.

\section{Results}

Out of 4,140 COVID-19 patients (55\% women, median age 52 years (interquartile range: $36-65)$ ), two thirds ( $N: 2,759)$ were outpatients, including 795 (19\% of the COVID-19 patients) who were evaluated in an outpatient emergency department. Of the 1,194 inpatients, 619 (15\% of the COVID-19 patients) were admitted to a Partners hospital but not to the ICU, while 575 (13.9\%) were admitted to the ICU, and 187 (4.5\%) died in-hospital. Older age, male sex, and comorbidities were more prevalent according to COVID-19 severity (Table). Prevalence of current/ever smoking was greater by severity ( $p$-value $<2.2 e-$ 16), and this group accounted for $58.3 \%$ of the deceased patients. Median household income per zip code had nonlinear association with severity. Table shows comorbidities and medication use according to severity. The most prevalent comorbidities among deceased patients were hyperlipidemia, hypertension, prior pneumonia ( $p$-values $<2.2 \mathrm{e}-16$ from the test of trend). Multiple other comorbidities also tracked with the disease severity. Use of angiotensin converting enzyme inhibitors or angiotensin receptor blockers was also greater by severity, but so was use of cardiovascular and other medications 
medRxiv preprint doi: https://doi.org/10.1101/2020.07.27.20163071; this version posted August 4, 2020. The copyright holder for this preprint (which was not certified by peer review) is the author/funder, who has granted medRxiv a license to display the preprint in perpetuity.

All rights reserved. No reuse allowed without permission.

including other antihypertensives, statins, antiplatelets, direct oral anticoagulants, antidiabetic medications and vitamin D3.

\section{Discussion}

In this large greater Boston study of 4,140 patients, multiple comorbidities and greater use of medications tracked with the severity of COVID-19. The data sources used in this study were limited to the use of the EHR. We found that prior use of antihypertensive medications as well as lipid lowering and other cardiovascular drugs such as direct oral anticoagulants and antiplatelets all tracked with increased severity of COVID-19 and should be further investigated with appropriate adjustment for confounders $^{5}$. Common prior comorbidities including hyperlipidemia, hypertension, and pneumonia also tracked with increased severity. 


\section{References}

1. Centers for Disease Control and Prevention; Coronavirus Disease 2019 (COVID-19). Accessed May 1, 2020. https://www.cdc.gov/coronavirus/2019-ncov/cases-updates/cases-in-us.html.

2. Estiri, H. and Murphy, S.N. Semi-Supervised Encoding for Outlier Detection in Clinical Observation Data. Comput Methods Programs in Biomed. 2019 Nov;181(1):1-16. doi:

10.1016/j.cmpb.2019.01.002.

3. Nalichowski, R., Keogh, D., Chueh, H. C., Murphy, S. N. (2006). Calculating the Benefits of a Research Patient Data Repository. AMIA Annual Symposium proceedings, 2006: 1044-1044.

4. Yu S, Ma Y, Gronsbell J, Cai T, et al. Enabling Phenotypic Big Data with PheNorm. J Am Med Inform Assoc. 2018;25(1):54-60. doi:10.1093/jamia/ocx111.

5. Mehta, N., Kalra, A., Nowacki, A.S., Anjewierden, S., Han, Z., Bhat, P., Carmona-Rubio, A.E., Jacob, M., Procop, G.W., Harrington, S. and Milinovich, A., 2020. Association of use of angiotensinconverting enzyme inhibitors and angiotensin II receptor blockers with testing positive for coronavirus disease 2019 (COVID-19). JAMA cardiology. 2020 May 5. doi:10.1001/jamacardio.2020.1855 
medRxiv preprint doi: https://doi.org/10.1101/2020.07.27.20163071; this version posted August 4, 2020. The copyright holder for this preprint (which was not certified by peer review) is the author/funder, who has granted medRxiv a license to display the preprint in perpetuity.

All rights reserved. No reuse allowed without permission.

\section{Footnotes:}

Authors of this manuscript have no conflicts of interest to declare.

\section{Funding}

This work was supported by the National Heart Lung and Blood Institute [T32 HL007575 to H.D., R01

HL134811; K24 HL136852; and HL 117861 to S.M., 5K01HL135342 to O.D.], and by philanthropic support from the Brigham COVID fund.

Corresponding Author:

Olga Demler odemler@bwh.harvard.edu 
medRxiv preprint doi: https://doi.org/10.1101/2020.07.27.20163071; this version posted August 4, 2020. The copyright holder for this preprint (which was not certified by peer review) is the author/funder, who has granted medRxiv a license to display the preprint in perpetuity. All rights reserved. No reuse allowed without permission.

Table. Demographic characteristics, clinical measurements, prior conditions and medication use by severity of COVID-191.

\begin{tabular}{|c|c|c|c|c|c|c|}
\hline & $\begin{array}{l}\text { Outpatient } \\
N=1964\end{array}$ & $\begin{array}{l}\text { Emergency } \\
\text { Department } \\
\mathrm{N}=795\end{array}$ & $\begin{array}{l}\text { Inpatient; } \\
\text { Non-ICU } \\
\mathrm{N}=619\end{array}$ & $\begin{array}{l}\text { Inpatient; } \\
\text { ICU } \\
\text { N=575 }\end{array}$ & $\begin{array}{l}\text { Deceased } \\
\mathrm{N}=187\end{array}$ & p-value ${ }^{1}$ \\
\hline Age median (IQR), y & $\begin{array}{l}47.0(32- \\
59)\end{array}$ & $43(31-55)$ & $62(48-76)$ & $62(50-72)$ & $81(73-87)$ & $5.59 e-153$ \\
\hline Female N (\%) & $\begin{array}{l}1243 \\
(63.3)\end{array}$ & $441(55.5)$ & $308(49.8)$ & $246(42.8)$ & $72(38.5)$ & $<2.2 \mathrm{e}-16$ \\
\hline Race/ethnicity N (\%) & & & & & & \\
\hline White & $969(49.3)$ & 301 (37.9) & $279(45.0)$ & $311(54.1)$ & $130(69.5)$ & $2.98 e-04$ \\
\hline Black & $322(16.4)$ & $138(17.4)$ & $119(19.2)$ & $80(13.9)$ & $31(16.6)$ & $7.15 e-01$ \\
\hline Hispanic & $221(11.2)$ & $132(16.6)$ & $72(11.6)$ & $52(9.0)$ & $11(5.9)$ & $3.44 \mathrm{e}-02$ \\
\hline Asian & $53(2.7)$ & $31(3.9)$ & $22(3.5)$ & $23(4.0)$ & $3(1.6)$ & $3.85 e-01$ \\
\hline $\begin{array}{l}\text { Other/ Not } \\
\text { recorded }\end{array}$ & 399 (20.3) & $193(24.3)$ & $127(20.5)$ & 109 (19.0) & $12(6.4)$ & \\
\hline Smoking N (\%) & & & & & & \\
\hline Current/ever & $440(22.4)$ & $160(20.2)$ & $239(38.6)$ & 229 (39.9) & $109(58.3)$ & $<2.2 \mathrm{e}-16$ \\
\hline Never & $\begin{array}{l}1118 \\
(56.9)\end{array}$ & $423(53.2)$ & $292(47.2)$ & $248(43.1)$ & $56(29.9)$ & $<2.2 \mathrm{e}-16$ \\
\hline Unknown & $406(20.7)$ & $212(26.7)$ & $88(14.2)$ & $98(17.0)$ & $22(11.8)$ & \\
\hline $\begin{array}{l}\text { Median household } \\
\text { income per zip code, } \\
\text { median (IQR), K\$ }\end{array}$ & $\begin{array}{l}68.2 \text { (53.3- } \\
97.5)\end{array}$ & $\begin{array}{l}64.2 \text { (53.2- } \\
90.8)\end{array}$ & $\begin{array}{l}67.6(54.4- \\
96.9)\end{array}$ & $\begin{array}{l}64.8(53.3- \\
85.3)\end{array}$ & $\begin{array}{l}68.3(55.0- \\
100.9)\end{array}$ & $1.10 \mathrm{e}-06$ \\
\hline
\end{tabular}


medRxiv preprint doi: https://doi.org/10.1101/2020.07.27.20163071; this version posted August 4, 2020. The copyright holder for this preprint (which was not certified by peer review) is the author/funder, who has granted medRxiv a license to display the preprint in perpetuity. All rights reserved. No reuse allowed without permission.

\begin{tabular}{|c|c|c|c|c|c|c|}
\hline $\begin{array}{l}\text { Max LOS in hospital } \\
\text { median (IQR), days }\end{array}$ & . & . & $\begin{array}{l}5.9(3.1- \\
10.4)\end{array}$ & $\begin{array}{l}13.3(6.2- \\
20.2)\end{array}$ & $\begin{array}{l}5.9(3.6- \\
9.9)\end{array}$ & $1.42 \mathrm{e}-08$ \\
\hline $\begin{array}{l}\text { Max LOS in intensive } \\
\text { care unit median } \\
\text { (IQR), days }\end{array}$ & . & . & . & $\begin{array}{l}7.7(3.8- \\
14.9)\end{array}$ & $\begin{array}{l}5.1(2.7- \\
8.7)\end{array}$ & $7.08 \mathrm{e}-06$ \\
\hline \multicolumn{7}{|c|}{ Clinical measurements at time of initial positive test ${ }^{2}$} \\
\hline $\begin{array}{l}\text { Temperature } \\
\text { median (IQR), } \mathrm{F}^{\circ}\end{array}$ & $\begin{array}{r}98.5 \text { (97.9- } \\
99.2) \\
(\mathrm{N}: 737)\end{array}$ & $\begin{array}{r}98.6(98.0- \\
99.3) \\
(\mathrm{N}: 765)\end{array}$ & $\begin{array}{r}98.6(97.8- \\
99.6) \\
(\mathrm{N}: 618)\end{array}$ & $\begin{array}{l}99.1(98.2- \\
100.3) \\
(N: 575)\end{array}$ & $\begin{array}{l}98.8(98.0- \\
100.5) \\
(N: 187)\end{array}$ & $2.72 \mathrm{E}-10$ \\
\hline $\begin{array}{l}\text { Systolic blood } \\
\text { pressure, } \mathrm{mm} \mathrm{Hg}\end{array}$ & $\begin{array}{r}139 \text { (114- } \\
146.5) \\
(\mathrm{N}: 31)\end{array}$ & $\begin{array}{r}134 \text { (121- } \\
148) \\
(\mathrm{N}: 737)\end{array}$ & $\begin{array}{r}132 \text { (117- } \\
146) \\
(\mathrm{N}: 615)\end{array}$ & $\begin{array}{l}135(119- \\
150) \\
(N: 571)\end{array}$ & $\begin{array}{l}126(108- \\
147) \\
(\mathrm{N}: 187)\end{array}$ & $3.62 \mathrm{e}-02$ \\
\hline $\begin{array}{l}\text { Diastolic blood } \\
\text { pressure, mm Hg }\end{array}$ & $\begin{array}{r}80(68-87) \\
(N: 31)\end{array}$ & $\begin{array}{r}80(72-89) \\
(N: 737)\end{array}$ & $\begin{array}{r}74(65-83) \\
(N: 615)\end{array}$ & $\begin{array}{l}74(65-83) \\
(N: 571)\end{array}$ & $\begin{array}{l}67 \text { (59.5- } \\
77.5) \\
(\mathrm{N}: 187)\end{array}$ & $5.67 e-23$ \\
\hline $\begin{array}{l}\text { Calculated mean } \\
\text { arterial pressure, } \\
\mathrm{mm} \mathrm{Hg}\end{array}$ & $\begin{array}{l}97.3(85.0- \\
106.8) \\
(\mathrm{N}: 31)\end{array}$ & $\begin{array}{l}98.3(89.0- \\
108.0) \\
(\mathrm{N}: 737)\end{array}$ & $\begin{array}{l}92.7(84.0- \\
103.7) \\
(\mathrm{N}: 617)\end{array}$ & $\begin{array}{l}94.7(84.7- \\
104.8) \\
(N: 575)\end{array}$ & $\begin{array}{l}88.0(76.5- \\
101.5) \\
(\mathrm{N}: 187)\end{array}$ & $3.89 e-01$ \\
\hline $\mathrm{BMI}, \mathrm{Kg} / \mathrm{m}^{2}$ & $\begin{array}{r}32.5(27.3- \\
36.4)(\mathrm{N}: \\
13)\end{array}$ & $\begin{array}{r}28.7(24.8- \\
33.2)(\mathrm{N}: \\
269)\end{array}$ & $\begin{array}{r}29.1(25.2- \\
33.6)(\mathrm{N}: \\
604)\end{array}$ & $\begin{array}{l}30.0(26.2- \\
34.0)(\mathrm{N}: \\
567)\end{array}$ & $\begin{array}{l}27.6(24.2- \\
32.2)(\mathrm{N}: \\
176)\end{array}$ & $9.08 \mathrm{e}-01$ \\
\hline $\begin{array}{l}\text { O2 Flow Rate } \\
\text { (L/min) }\end{array}$ & $\begin{array}{r}2(2-4) \\
(N: 5)\end{array}$ & $\begin{array}{r}2(2-2.5) \\
(N: 19)\end{array}$ & $\begin{array}{l}2(2-2) \\
(N: 420)\end{array}$ & $\begin{array}{l}2.0(2-4) \\
(N: 478)\end{array}$ & $\begin{array}{l}3.0(2-6) \\
(N: 167)\end{array}$ & $4.64 \mathrm{e}-04$ \\
\hline O2 Device $(\mathrm{N})$ & & & & & & \\
\hline $\begin{array}{l}\text { None (Room } \\
\text { air) }\end{array}$ & $26(1.3)$ & 671 (84.4) & $512(82.7)$ & $414(72.0)$ & 82 (43.9) & $<2.2 \mathrm{e}-16$ \\
\hline
\end{tabular}


medRxiv preprint doi: https://doi.org/10.1101/2020.07.27.20163071; this version posted August 4, 2020. The copyright holder for this preprint (which was not certified by peer review) is the author/funder, who has granted medRxiv a license to display the preprint in perpetuity. All rights reserved. No reuse allowed without permission.

\begin{tabular}{|c|c|c|c|c|c|c|}
\hline Nasal cannula & $1(0.1)$ & $8(1.0)$ & $101(16.3)$ & $98(17.0)$ & $51(27.3)$ & $<2.2 \mathrm{e}-16$ \\
\hline $\begin{array}{l}\text { Non-rebreather } \\
\text { mask }\end{array}$ & 0 & 0 & $2(0.3)$ & $31(5.4)$ & $37(19.8)$ & $<2.2 \mathrm{e}-16$ \\
\hline Other devices & $1(0.1)$ & 0 & $2(0.3)$ & $32(5.6)$ & $16(8.6)$ & . \\
\hline SpO2 median (IQR) & $\begin{array}{r}98(96-98) \\
(N: 53)\end{array}$ & $\begin{array}{r}98 \text { (97-99) } \\
(\mathrm{N}: 755)\end{array}$ & $\begin{array}{r}96(94-98) \\
(N: 616)\end{array}$ & $\begin{array}{l}96(93-97) \\
(N: 569)\end{array}$ & $\begin{array}{l}95(92-97) \\
(\mathrm{N}: 187)\end{array}$ & $2.52 \mathrm{e}-46$ \\
\hline $\begin{array}{l}\text { Glasgow Coma Scale } \\
\text { Score }\end{array}$ & $\begin{array}{r}15(15-15) \\
(\mathrm{N}: 7)\end{array}$ & $\begin{array}{r}15(15-15) \\
(\mathrm{N}: 293)\end{array}$ & $\begin{array}{r}15(15-15) \\
(N: 428)\end{array}$ & $\begin{array}{l}15(14-15) \\
(N: 507)\end{array}$ & $\begin{array}{l}14(9.5-15) \\
(\mathrm{N}: 138)\end{array}$ & $1.92 \mathrm{e}-38$ \\
\hline $\begin{array}{l}\text { Have you had a } \\
\text { fever or felt feverish } \\
\text { in the past } 24 \\
\text { hours? }\end{array}$ & & & & & & \\
\hline Yes & $10(0.51)$ & 344 (43.27) & $\begin{array}{r}356 \\
(57.51)\end{array}$ & $\begin{array}{l}376 \\
(65.39)\end{array}$ & $95(50.80)$ & $<2.2 \mathrm{e}-16$ \\
\hline No & $14(0.71)$ & $235(29.56)$ & $\begin{array}{r}215 \\
(34.73)\end{array}$ & $\begin{array}{l}146 \\
(25.39)\end{array}$ & $51(27.27)$ & $<2.2 \mathrm{e}-16$ \\
\hline Unknown & $\begin{array}{r}1940 \\
(98.83)\end{array}$ & $216(27.17)$ & $48(7.75)$ & $53(9.22)$ & $41(21.93)$ & . \\
\hline $\begin{array}{l}\text { Do you have trouble } \\
\text { breathing or a new } \\
\text { cough? }\end{array}$ & & & & & & \\
\hline Yes & $8(0.41)$ & $398(50.06)$ & $\begin{array}{r}374 \\
(60.42)\end{array}$ & $\begin{array}{l}399 \\
(69.39)\end{array}$ & $98(52.41)$ & $<2.2 \mathrm{e}-16$ \\
\hline No & $15(0.76)$ & $185(23.27)$ & $\begin{array}{r}205 \\
(33.12)\end{array}$ & $\begin{array}{l}123 \\
(21.39)\end{array}$ & 43 (22.99) & $<2.2 \mathrm{e}-16$ \\
\hline
\end{tabular}


medRxiv preprint doi: https://doi.org/10.1101/2020.07.27.20163071; this version posted August 4, 2020. The copyright holder for this preprint (which was not certified by peer review) is the author/funder, who has granted medRxiv a license to display the preprint in perpetuity. All rights reserved. No reuse allowed without permission.

\begin{tabular}{|c|c|c|c|c|c|c|}
\hline Unknown & $\begin{array}{r}1941 \\
(98.83)\end{array}$ & $212(26.67)$ & $40(6.46)$ & $53(9.22)$ & $46(24.60)$ & \\
\hline \multicolumn{7}{|l|}{ Prior Conditions $^{3}$} \\
\hline Pneumonia & $\begin{array}{l}366 \\
(18.64)\end{array}$ & 147 (18.49) & $\begin{array}{l}217 \\
(35.06)\end{array}$ & $\begin{array}{l}164 \\
(28.52)\end{array}$ & 97 (51.87) & $<2.2 \mathrm{e}-16$ \\
\hline Hyperlipidemia & $\begin{array}{l}598 \\
(30.45)\end{array}$ & 196 (24.65) & $\begin{array}{l}324 \\
(52.34)\end{array}$ & $\begin{array}{l}284 \\
(49.39)\end{array}$ & $\begin{array}{l}128 \\
(68.45)\end{array}$ & $<2.2 \mathrm{e}-16$ \\
\hline Hypertension & $\begin{array}{l}318 \\
(16.19)\end{array}$ & 94 (11.82) & $\begin{array}{l}209 \\
(33.76)\end{array}$ & $\begin{array}{l}183 \\
(31.83)\end{array}$ & $\begin{array}{l}106 \\
(56.68)\end{array}$ & $<2.2 \mathrm{e}-16$ \\
\hline $\begin{array}{l}\text { Heart valve } \\
\text { disorders }\end{array}$ & $143(7.28)$ & $43(5.41)$ & $\begin{array}{l}100 \\
(16.16)\end{array}$ & 81 (14.09) & 66 (35.29) & $<2.2 \mathrm{e}-16$ \\
\hline Atrial fibrillation & $40(2.04)$ & $6(0.75)$ & $39(6.30)$ & $33(5.74)$ & $30(16.04)$ & $<2.2 \mathrm{e}-16$ \\
\hline $\begin{array}{l}\text { Pulmonary heart } \\
\text { disease }\end{array}$ & 47 (2.39) & $14(1.76)$ & $50(8.08)$ & $29(5.04)$ & $24(12.83)$ & 5.637e-14 \\
\hline $\begin{array}{l}\text { Deep vein } \\
\text { thrombosis }\end{array}$ & $31(1.58)$ & $6(0.75)$ & $29(4.68)$ & $15(2.61)$ & $13(6.95)$ & $1.092 \mathrm{e}-06$ \\
\hline $\begin{array}{l}\text { Other } \\
\text { Cardiovascular } \\
\text { Disease }^{4}\end{array}$ & $146(7.43)$ & $44(5.53)$ & $\begin{array}{l}107 \\
(17.29)\end{array}$ & $\begin{array}{l}113 \\
(19.65)\end{array}$ & 55 (29.41) & $<2.2 \mathrm{e}-16$ \\
\hline Alzheimer's disease & $20(1.02)$ & $1(0.13)$ & $13(2.10)$ & $7(1.22)$ & $8(4.28)$ & $2.28 \mathrm{e}-03$ \\
\hline Diabetes mellitus & & & & & & \\
\hline Type II & $140(7.13)$ & $39(4.91)$ & $\begin{array}{l}103 \\
(16.64)\end{array}$ & $\begin{array}{l}106 \\
(18.43)\end{array}$ & $55(29.41)$ & $<2.2 \mathrm{e}-16$ \\
\hline Type I & $6(0.31)$ & $5(0.63)$ & $4(0.65)$ & $7(1.22)$ & $0(0.00)$ & $8.71 e-02$ \\
\hline Obesity & $\begin{array}{l}505 \\
(25.71)\end{array}$ & $186(23.40)$ & $\begin{array}{l}216 \\
(34.89)\end{array}$ & $\begin{array}{l}171 \\
(29.74)\end{array}$ & $57(30.48)$ & $6.05 e-04$ \\
\hline
\end{tabular}


medRxiv preprint doi: https://doi.org/10.1101/2020.07.27.20163071; this version posted August 4, 2020. The copyright holder for this preprint (which was not certified by peer review) is the author/funder, who has granted medRxiv a license to display the preprint in perpetuity. All rights reserved. No reuse allowed without permission.

\begin{tabular}{|c|c|c|c|c|c|c|}
\hline $\begin{array}{l}\text { Cancer (breast, } \\
\text { prostate, thyroid) }\end{array}$ & $59(3.00)$ & $32(2.64)$ & $44(7.11)$ & $28(4.87)$ & $28(14.97)$ & $1.818 \mathrm{e}-10$ \\
\hline $\begin{array}{l}\text { Tobacco use } \\
\text { disorder }\end{array}$ & $\begin{array}{l}245 \\
(12.47)\end{array}$ & $94(11.82)$ & $\begin{array}{l}159 \\
(25.69)\end{array}$ & $\begin{array}{l}148 \\
(25.74)\end{array}$ & $82(43.85)$ & $<2.2 \mathrm{e}-16$ \\
\hline Alcoholism & $76(3.87)$ & 39 (4.91) & $72(11.63)$ & $36(6.26)$ & $16(8.56)$ & $4.846 e-07$ \\
\hline Asthma & $\begin{array}{l}235 \\
(11.97)\end{array}$ & $102(12.83)$ & $90(14.54)$ & 81 (14.09) & $25(13.37)$ & $9.74 \mathrm{e}-02$ \\
\hline Osteoarthrosis & $\begin{array}{l}294 \\
(14.97)\end{array}$ & $98(12.33)$ & $\begin{array}{l}179 \\
(28.92)\end{array}$ & $\begin{array}{l}129 \\
(22.43)\end{array}$ & $70(37.43)$ & $<2.2 \mathrm{e}-16$ \\
\hline Osteoporosis & $112(5.70)$ & $26(3.27)$ & $77(12.44)$ & $47(8.17)$ & $42(22.46)$ & $6.395 e-14$ \\
\hline $\begin{array}{l}\text { Gastroesophageal } \\
\text { reflux disease }\end{array}$ & $\begin{array}{l}497 \\
(25.31)\end{array}$ & $180(22.64)$ & $\begin{array}{l}216 \\
(34.89)\end{array}$ & $\begin{array}{l}167 \\
(29.04)\end{array}$ & $78(41.71)$ & $2.403 \mathrm{e}-07$ \\
\hline $\begin{array}{l}\text { Diverticulosis and } \\
\text { diverticulitis }\end{array}$ & $147(7.48)$ & $56(7.04)$ & $\begin{array}{l}111 \\
(17.93)\end{array}$ & $89(15.48)$ & $47(25.13)$ & $<2.2 \mathrm{e}-16$ \\
\hline Renal failure & 38 (1.93) & $12(1.51)$ & 73 (11.79) & $72(12.52)$ & $54(28.88)$ & $<2.2 \mathrm{e}-16$ \\
\hline Abdominal hernia & $152(7.74)$ & $62(7.80)$ & $98(15.83)$ & $74(12.87)$ & 37 (19.79) & $7.591 \mathrm{e}-12$ \\
\hline Insomnia & $162(8.25)$ & $68(8.55)$ & $78(12.60)$ & 70 (12.17) & $30(16.04)$ & $5.046 e-06$ \\
\hline $\begin{array}{l}\text { Obstructive sleep } \\
\text { apnea }\end{array}$ & 91 (4.63) & $41(5.16)$ & $44(7.11)$ & 58 (10.09) & 25 (13.37) & $6.146 e-10$ \\
\hline Prior Medications & & & & & & \\
\hline $\begin{array}{l}\text { Angiotensin } \\
\text { converting enzyme } \\
\text { inhibitors }\end{array}$ & $\begin{array}{l}270 \\
(13.75)\end{array}$ & 85 (10.69) & $\begin{array}{l}162 \\
(26.17)\end{array}$ & $\begin{array}{l}159 \\
(27.65)\end{array}$ & $80(42.78)$ & $2.2 \mathrm{e}-16$ \\
\hline $\begin{array}{l}\text { Angiotensin } \\
\text { receptor blockers }\end{array}$ & $128(6.52)$ & $35(4.40)$ & $76(12.28)$ & $71(12.35)$ & $38(20.32)$ & $1.129 \mathrm{e}-14$ \\
\hline
\end{tabular}


medRxiv preprint doi: https://doi.org/10.1101/2020.07.27.20163071; this version posted August 4, 2020. The copyright holder for this preprint (which was not certified by peer review) is the author/funder, who has granted medRxiv a license to display the preprint in perpetuity. All rights reserved. No reuse allowed without permission.

\begin{tabular}{|c|c|c|c|c|c|c|}
\hline Beta blockers & $\begin{array}{l}353 \\
(17.97)\end{array}$ & 111 (13.96) & $\begin{array}{l}223 \\
(36.03)\end{array}$ & $\begin{array}{l}192 \\
(33.39)\end{array}$ & $\begin{array}{l}119 \\
(63.64)\end{array}$ & $<2.2 \mathrm{e}-16$ \\
\hline $\begin{array}{l}\text { Calcium channel } \\
\text { blockers }\end{array}$ & $\begin{array}{l}254 \\
(12.93)\end{array}$ & 73 (9.18) & $\begin{array}{l}154 \\
(24.88)\end{array}$ & $\begin{array}{l}147 \\
(25.57)\end{array}$ & $81(43.32)$ & $<2.2 \mathrm{e}-16$ \\
\hline Diuretics & $\begin{array}{l}281 \\
(14.31)\end{array}$ & 85 (10.69) & $\begin{array}{l}198 \\
(31.99)\end{array}$ & $\begin{array}{l}180 \\
(31.30)\end{array}$ & $\begin{array}{l}115 \\
(61.50)\end{array}$ & $<2.2 \mathrm{e}-16$ \\
\hline $\begin{array}{l}\text { Mineralocorticoid } \\
\text { receptor } \\
\text { antagonists }\end{array}$ & $54(2.75)$ & $18(2.26)$ & 21 (3.39) & $22(3.83)$ & $15(8.02)$ & $1.59 \mathrm{e}-03$ \\
\hline $\begin{array}{l}\text { Other } \\
\text { antihypertensive } \\
\text { medications }^{5}\end{array}$ & 97 (4.94) & $43(5.41)$ & 95 (15.35) & $86(14.96)$ & $40(21.39)$ & $<2.2 \mathrm{e}-16$ \\
\hline Statins & $\begin{array}{l}449 \\
(22.86)\end{array}$ & $148(18.62)$ & $\begin{array}{l}305 \\
(49.27)\end{array}$ & $\begin{array}{l}282 \\
(49.04)\end{array}$ & $\begin{array}{l}132 \\
(70.59)\end{array}$ & $<2.2 \mathrm{e}-16$ \\
\hline Heparins & $189(9.62)$ & 70 (8.81) & $\begin{array}{l}189 \\
(30.53)\end{array}$ & $\begin{array}{l}125 \\
(21.74)\end{array}$ & $85(45.45)$ & $<2.2 \mathrm{e}-16$ \\
\hline $\begin{array}{l}\text { Oral anticoagulants } \\
6\end{array}$ & 96 (4.89) & $27(3.40)$ & 89 (14.38) & $66(11.48)$ & $58(31.02)$ & $<2.2 \mathrm{e}-16$ \\
\hline Platelet inhibitors & $\begin{array}{l}667 \\
(33.96)\end{array}$ & 254 (31.95) & $\begin{array}{l}346 \\
(55.90)\end{array}$ & $\begin{array}{l}310 \\
(53.91)\end{array}$ & $\begin{array}{l}140 \\
(74.87)\end{array}$ & $<2.2 \mathrm{e}-16$ \\
\hline Insulin & $135(6.87)$ & $58(7.30)$ & $\begin{array}{l}124 \\
(20.03)\end{array}$ & $\begin{array}{l}139 \\
(24.17)\end{array}$ & 71 (37.97) & $<2.2 \mathrm{e}-16$ \\
\hline Metformin & $\begin{array}{l}206 \\
(10.49)\end{array}$ & $81(10.19)$ & $\begin{array}{l}126 \\
(20.36)\end{array}$ & $\begin{array}{l}116 \\
(20.17)\end{array}$ & $42(22.46)$ & $3.267 e-16$ \\
\hline $\begin{array}{l}\text { Other antidiabetic } \\
\text { medications }^{7}\end{array}$ & $160(8.15)$ & $53(6.67)$ & $\begin{array}{l}102 \\
(16.48)\end{array}$ & $\begin{array}{l}107 \\
(18.61)\end{array}$ & $58(31.02)$ & $<2.2 \mathrm{e}-16$ \\
\hline
\end{tabular}


medRxiv preprint doi: https://doi.org/10.1101/2020.07.27.20163071; this version posted August 4, 2020. The copyright holder for this preprint (which was not certified by peer review) is the author/funder, who has granted medRxiv a license to display the preprint in perpetuity.

All rights reserved. No reuse allowed without permission.

$\begin{array}{lllllll}\text { Cholecalciferol } & 469 & 137(17.23) & 236 & 161 & 102 & <2.2 \mathrm{e}-16 \\ (\text { vitamin D3) } & (23.88) & & (38.13) & (28.00) & (54.55) & \end{array}$

${ }_{1}^{1}$ P-values are reported for Chi-square tests of trend in proportions of all categorical variables and for tests of trend in the means for all continuous variables. Test of the difference in means was used to compare median income across severity categories. ${ }^{2}$ Clinical measurements based on EHR records of patients on the date of their first COVID-19 positive test, or the closest measures within 2 weeks of the positive test. ${ }^{3}$ Diagnosis and medications that were recorded for less than $10 \%$ of the patients were not reported in this table. Comorbid conditions were computed by Partners' HealthCare System by combining the ICD-10 codes with Natural Language Processing of medical notes using Machine Learning methods and were validated by medical professionals. Asthma diagnosis was calculated using ICD-10 billing codes. ${ }^{4}$ Other cardiovascular disease represent the total number of patients with records of aortic aneurysm, atrioventricular block, coronary atherosclerosis, heart valve disorders, ischemic stroke, myocardial infarction, peripheral vascular disease. ${ }^{5}$ Renin inhibitors, pulmonary anti-hypertensives, angiotensin receptor-neprilysin inhibitors. ${ }^{6}$ Direct oral anticoagulants or warfarin. ${ }^{7}$ Dipeptidyl peptidase 4 inhibitors, sodium glucose co-transporter 2 inhibitors, glucagon-like peptide 1 agonists, sulfonylureas, thiazolidinediones. 